\title{
Letter: Multiple renal vessels associated with testicular vessels
}

\author{
Mohammadali M. Shoja $\cdot$ Marios Loukas • \\ Jalal Etemadi $\cdot$ R. Shane Tubbs
}

Received: 24 February 2011 / Accepted: 28 March 2011 / Published online: 9 April 2011

(C) Springer-Verlag 2011

We read with great interest the recent paper published by Naito et al. [1] entitled "Multiple renal vessels associated with testicular vessels." The authors reported five right renal arteries and three left renal arteries arising from the abdominal aorta; both right and left gonadal arteries originated from one of the multiple ipsilateral renal arteries. The report is interesting; however, we would like to add that the statistical proof for the tendency of gonadal arteries to originate from kidneys that possess an accessory arterial supply was first described by our group in a series of 50 healthy individuals in 2007 [2]. In our previous study, we examined angiographic images of 98 kidneys; $39 \%$ of the kidneys had at least one accessory renal artery. On 14 sides, the gonadal artery originated from the main or accessory renal artery. Ten out of fourteen kidneys with an aberrant gonadal artery had an associated accessory renal artery. There was a statistically significant association between aberrant renal origin of the gonadal artery and the presence of multiple renal arteries $(P=0.007)$. We believe that the aberrant origin of the gonadal artery from the renal vasculature is an under-recognized variation.

\footnotetext{
M. M. Shoja ( $\square)$ · J. Etemadi

Tuberculosis and Lung Disease Research Center,

Tabriz University of Medical Sciences,

Golgasht Avenue, Tabriz, Iran

e-mail: shoja.m@gmail.com

M. Loukas

Department of Anatomical Sciences, School of Medicine,

St. George's University, Grenada, West Indies

R. Shane Tubbs

Section of Pediatric Neurosurgery,

Children's Hospital, Birmingham, AL, USA
}

Such data should be a relevant addition to the report of Naito et al. [1]. Furthermore, the bilateral origin of the gonadal artery in an individual with bilateral multiple renal arteries may be an additional proof to our hypothesis that a common embryonic error may underlie the formation of accessory renal and aberrant gonadal arteries.

Sincerely,

Mohammadali M. Shoja

Marios Loukas

Jalal Etemadi

R. Shane Tubbs

Conflict of interest None.

\section{References}

1. Naito M, Hirai S, Terayama H, Qu N, Yi SQ, Miyaki T, Itoh M (2011) Multiple renal vessels associated with testicular vessels. Surg Radiol Anat. doi:10.1007/s00276-011-0793-y

2. Shoja MM, Tubbs RS, Shakeri AB, Oakes WJ (2007) Origins of the gonadal artery: embryologic implications. Clin Anat 20:428-432 